\title{
Robotically Assembled Aerospace Structures: Digital Material Assembly using a Gantry-Type Assembler
}

\author{
Greenfield Trinh, Grace Copplestone, \\ Molly O'Connor, Steven Hu, \\ Sebastian Nowak, Kenneth Cheung \\ NASA Ames Research Center \\ Coded Structures Lab, MS 202-3 \\ Moffett Field, CA 94035 \\ greenfield.trinh@nasa.gov
}

\author{
Benjamin Jenett \\ Center for Bits and Atoms \\ Massachusetts Institute of Technology \\ Cambridge, MA \\ bej@mit.edu
}

\author{
Daniel Cellucci \\ Department of Mechanical \\ and Aerospace Engineering \\ Cornell University \\ Ithaca, NY \\ dwc238@cornell.edu
}

\begin{abstract}
This paper evaluates the development of automated assembly techniques for discrete lattice structures using a multiaxis gantry type CNC machine. These lattices are made of discrete components called "digital materials." We present the development of a specialized end effector that works in conjunction with the CNC machine to assemble these lattices. With this configuration we are able to place voxels at a rate of 1.5 per minute.
\end{abstract}

The scalability of digital material structures due to the incremental modular assembly is one of its key traits and an important metric of interest. We investigate the build times of a $5 \times 5$ beam structure on the scale of 1 meter ( 325 parts), 10 meters $(3,250$ parts $)$, and 30 meters $(9,750$ parts $)$. Utilizing the current configuration with a single end effector, performing serial assembly with a globally fixed feed station at the edge of the build volume, the build time increases according to a scaling law of $n^{4}$, where $\mathbf{n}$ is the build scale. Build times can be reduced significantly by integrating feed systems into the gantry itself, resulting in a scaling law of $n^{3}$. A completely serial assembly process will encounter time limitations as build scale increases.

Automated assembly for digital materials can assemble high performance structures from discrete parts, and techniques such as built in feed systems, parallelization, and optimization of the fastening process will yield much higher throughput.

\section{TABle of Contents}

1. INTRODUCTION............................

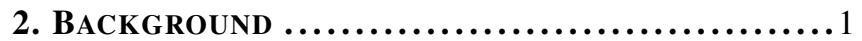

3. Methodology ............................

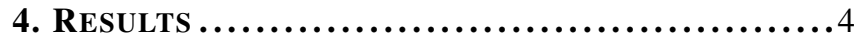

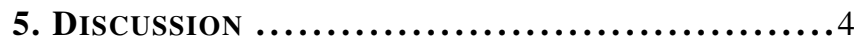

6. Conclusion $\ldots \ldots \ldots \ldots \ldots \ldots \ldots \ldots \ldots \ldots \ldots \ldots \ldots \ldots \ldots . \ldots \ldots$

ACKNOWLEDGMENTS ........................6

REFERENCES .............................6

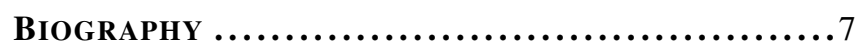

\section{INTRODUCTION}

We present the development of automated assembly of discrete lattice structures using a gantry-type robotic assembler. These structures, which are call digital materials, are defined as a discrete set of parts linked with a discrete set of relative positions and orientations [1]. The objective of this work is to evaluate applications of automated assembly using digital materials and to analyze the potential for scaling this system.

978-1-5090-1613-6/17/\$31.00 (c)2017 IEEE
Digital cellular structures are well suited for aerospace applications due to the ease at which high stiffness to weight ratios can be achieved at ultra-light mass densities, as well as reconfigurability and reusability [2] [3]. These materials can serve as primary mechanical structure for aerospace systems such as a wing [4], spacecraft structure, habitat, or other high performance structures [3]. Additionally, the modular and periodic nature of the structures can be leveraged to enable assembly automation.

This paper presents a brief overview of the literature of automated assembly and digital cellular materials followed by the methodology and results for automated assembly of digital materials using a gantry system. The discussion of performance metrics and characteristics of the digital material assembly system includes comparison to other in-space manufacturing systems.

\section{BACKGROUND}

Automated Assembly

In-space assembly (ISA) and assembly in factory settings are being revolutionized by robotic automation. The utilization of robots to automate assembly helps reduce errors, increase throughput efficiency, and ultimately reduce costs [5]. For the space industry, the capability to perform ISA greatly expands mission opportunities and equipment performance for scientists and explorers. The need to construct structures much larger than the launch volume of rockets requires further maturation of ISA techniques. The most significant example of ISA technology is the International Space Station (ISS) and further development of this technology will enable larger structures for new scientific and exploration capabilities such as a large aperture telescope and larger space stations. Current ISA systems rely on astronaut Extravehicular Activity (EVA) in conjunction with assistive automation technologies to perform assembly operations, but for many proposed large structure applications, this is not feasible [6]. In the future, full automation of assembly tasks may help reduce costs, reduce risk, and free up astronaut responsibilities to allow greater focus on science and exploration. We can leverage advances in robotic technology to perform these functions.

On-going assembly research includes assembly of erectable truss elements into structures for space telescopes [7]. One example of these techniques build upon EVA assembly techniques and involves welding of the truss elements using a jigging robot to align the elements. Another truss assembly system is called the Trusselator [8]. This system, manufactured by Tethers Unlimited, leverages carbon fiber 
composite thermoplastic technologies to create lightweight truss extrusions.

The automated assembly work we are conducting will provide important insight into how to scale digital material assembly systems to build large structures in space. An assembly system, similiar to the one shown in Figure 1 in conjunction with robots that traverse these lattice structures can help realize this idea [9].The current state of the art in digital material assembly, described herein, still requires human interaction with an explicit goal of fully automating these systems.

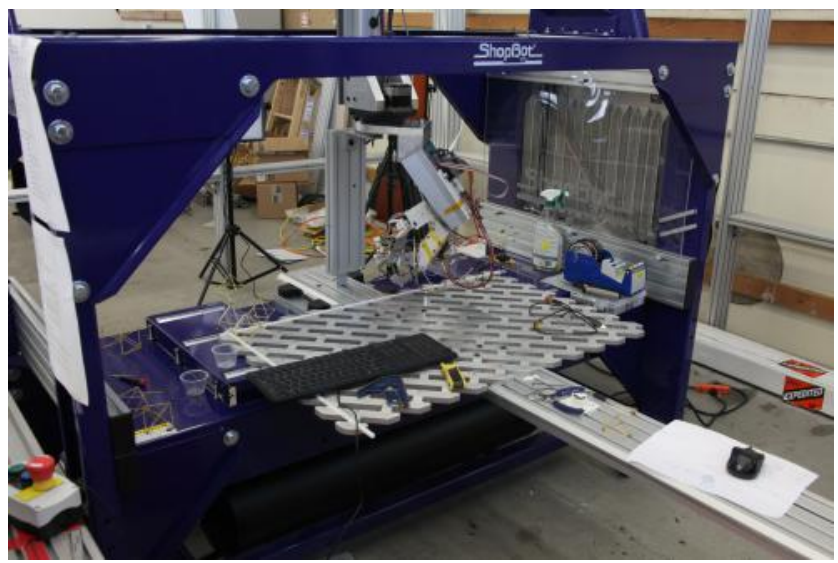

Figure 1. 5-axis CNC gantry machine being used for automated assembly of voxel lattices.

\section{Digital Cellular Material}

Digital cellular materials is a relative new field of study where the lattice based cellular structures are made using reversible joint connections. These lattice structures have a low relative mass density and can be made to achieve high stiffness and strength to weight ratios at ultralight mass densities [2] [4]. Different constituent materials and lattice geometries can affect the properties and performance of the material. They can therefore be "tuned" to applications as needed [10]. Common geometries that have been investigated include the Octet lattice and Kelvin lattice. Each element is referred to herein as a voxel, or volumetric pixel.

One of the key hurdles of space travel is the limited resources that are available [11] [12]. The concept of digital materials leverages reusability of components within the reversibility assembled structure to be able to reconfigure it to different uses [1]. We propose that digital cellular materials can serve as a base mechanical structural subsystem that is lightweight, stiff, and can be reconfigured depending on mission need. The periodic nature of most currently used lattice geometries make them well suited for robotic manipulation and assembly. Another potential of these materials is the scaling properties of the lattices. In conjunction with assembly robots we will be able to construct structures much larger than previously completed.

In order to utilize and manufacture these structures, an assembly system is needed for placement and joining of these components. The Coded Structures Lab at NASA Ames Research Center is conducting research on the development of a digital material ecosystem consisting of various lattice geometries, robots, and assembly systems that can manipulate and traverse through the structures, along with the software and support needed to realize the potential of these systems [9].

\section{Methodology}

Overview and Design Objectives

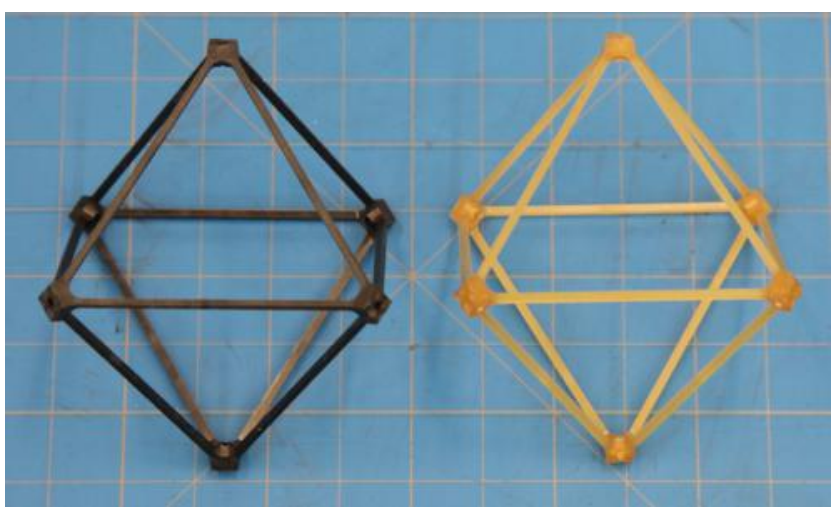

Figure 2. Image of carbon fiber filled (left) and glass-filled (right) PEI voxels.

The digital cellular lattice geometry assembled in this work is a cuboctohedra, or CubOct, lattice, as seen in Figure 2. The CubOct lattice is composed of discrete octahedral voxels consisting of 12 equal length struts [2]. Each voxel is 3 inches in its longest dimension. These voxels are injection molded structures that are made from different plastics including: Polyetherimide plastic (PEI), Glass filled PEI, and Carbon fiber filled PEI. For assembly testing purposes we utilize the $20 \%$ glass filled pieces due to their toughness. The nodes of the lattice are fastened together using 0-80 screws and nuts to a torque of 1 in-lb. The objective of this demonstration is to start with assembling rectangular prism structures and then expanding to larger beam structures.

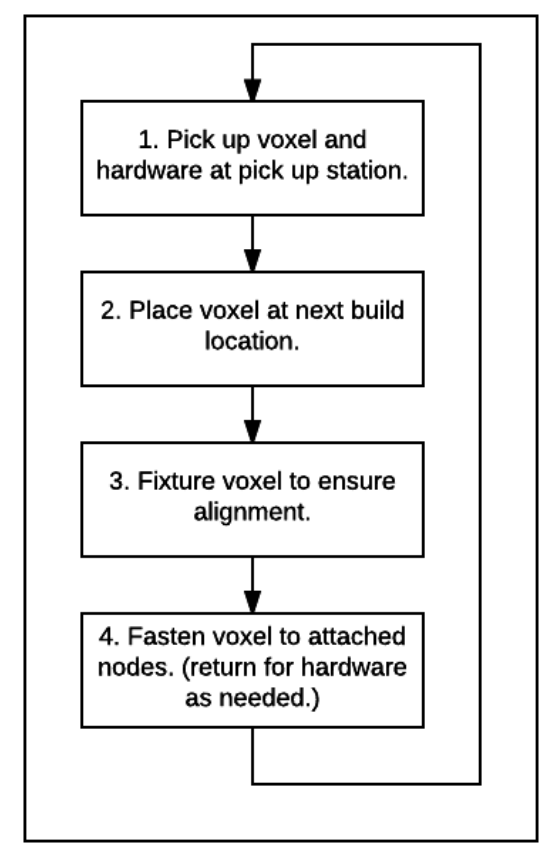

Figure 3. This flow chart describes the process for assembling each voxel. 


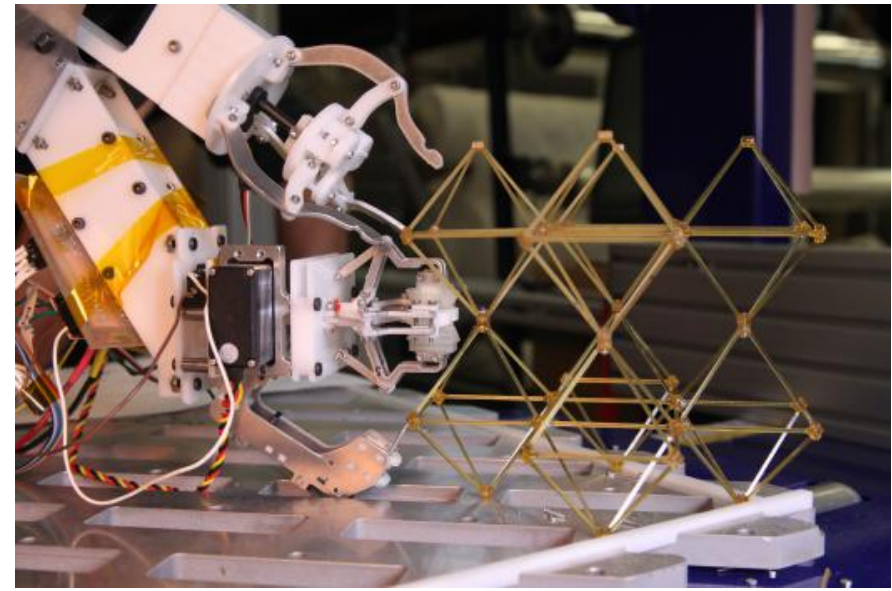

Figure 4. End effector performing placement and fastening of voxel.

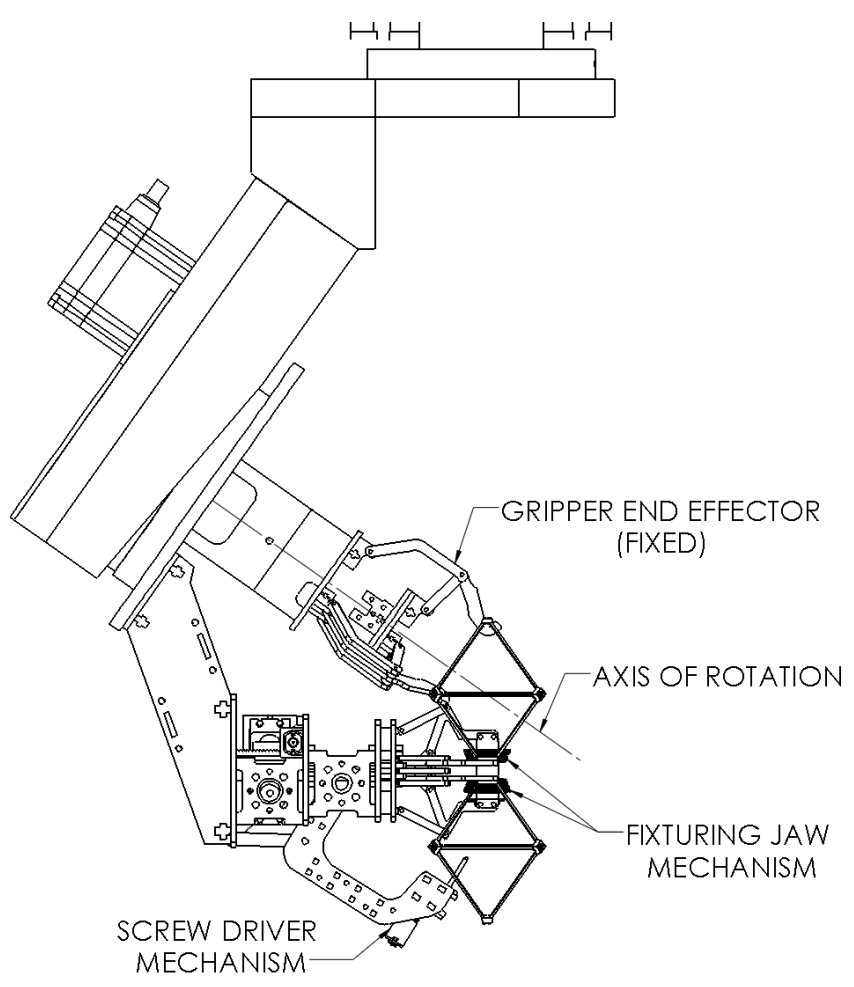

Figure 5. This is a diagram of the end effector.

To accomplish the task of assembling the discrete voxel structures, we utilize a commercial gantry style 5-axis CNC machine with a custom end effector. The CNC provides coordinate positioning to the end effector. The end effector performs retrieval of voxel and fastening hardware and then fixtures and fastens the voxel joints together. The build plate on the $\mathrm{CNC}$ machine is designed to to provide clearance for the end effector operations. Figure 3 provides a flow chart of the assembly process.

To test the system, we begin with construction of cube lattices at size $2 \times 2 \times 2$ and $3 \times 3 \times 3$. We then expand to rectangular prism structures of $2 \times 2 \times 12$ and $3 \times 3 \times 12$. We also test the build algorithm by testing structures with holes in them.

\section{Gantry and End Effector Design}

We use an architecture that consists of a pick-up end effector and a separate joining end effector mounted together on the system, as shown in Figure 4. The pick-up end effector is a linkage pick-up claw mechanism. The mechanism for fixturing the voxel nodes and fastening them are mounted on a separate unit that can rotate \pm 120 degrees to fasten the adjoining nodes of the placed voxel. A diagram of the end effector is shown in Figure 5. The end effectors are controlled by an Arduino microcontroller that interfaces with the CNC gantry outputs. The fixturing mechanism is designed to hold the voxel joints together as well as hold a nut and screw.

To ensure the joint is fastened to the correct torque, a feedback system that senses if the screwdriver has reached the correct torque is used. This is accomplished by sensing an over-current when the motor stalls. The motor stall torque needed, depending on material of the voxel, can be adjust in software. If the torque is not reached, the system performs the action again to ensure correct bolt torque.

The nut and bolt dispensing mechanisms, shown in Figure 6 , is located at the edge of the build volume. The end effector travels to the pick-up station for hardware as needed. The dispensing mechanisms provide the robot with one bolt and one nut at a time, but are able to store the extra parts inside a magazine that can be switched out quickly.

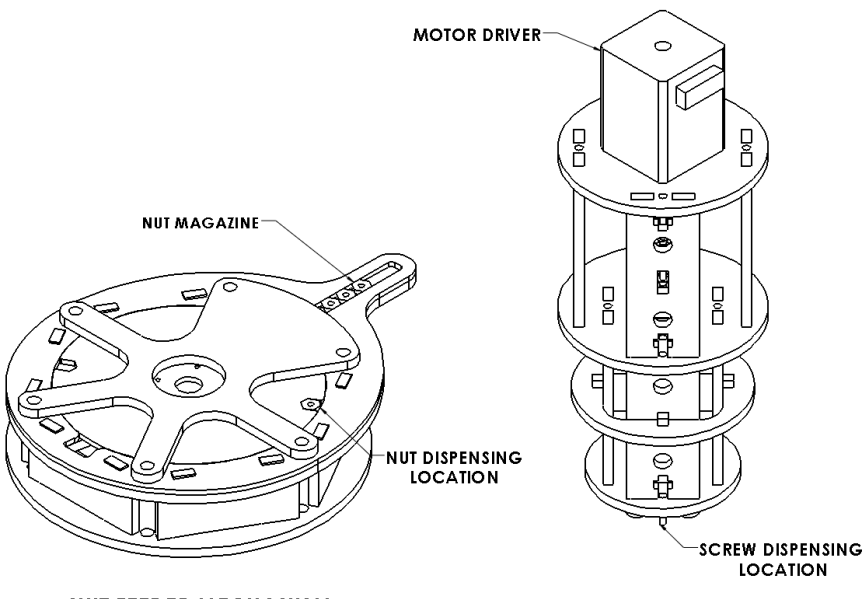

NUT FEEDER MECHANISM

SCREW FEEDER MECHANISM

\section{Figure 6. Nut and screw feed mechanism at pick up station.}

\section{Software for Path Planning and Visualization}

A 5-axis ShopBot is used in the development of an automated voxel structure build. For this, tool path and build instructions are needed. Software is written to interpret a voxel structure, determine its build path, and provide instructions to the machine.

Assembly of simple square voxel structures have been automated to date. This includes a $2 \times 2 \times 2$ and $3 \times 3 \times 3$ voxel cube. To expand on these simple structures, its desired to have a simple method to define complex voxel structures.

To describe the voxel structures in software, the current implementation is a 3-dimensional Boolean matrix defining the voxel structure. Adding a layer in $\mathrm{x}, \mathrm{y}$, or $\mathrm{z}$ directions is done by extending the matrix in $\mathrm{x}, \mathrm{y}$, or $\mathrm{z}$. 

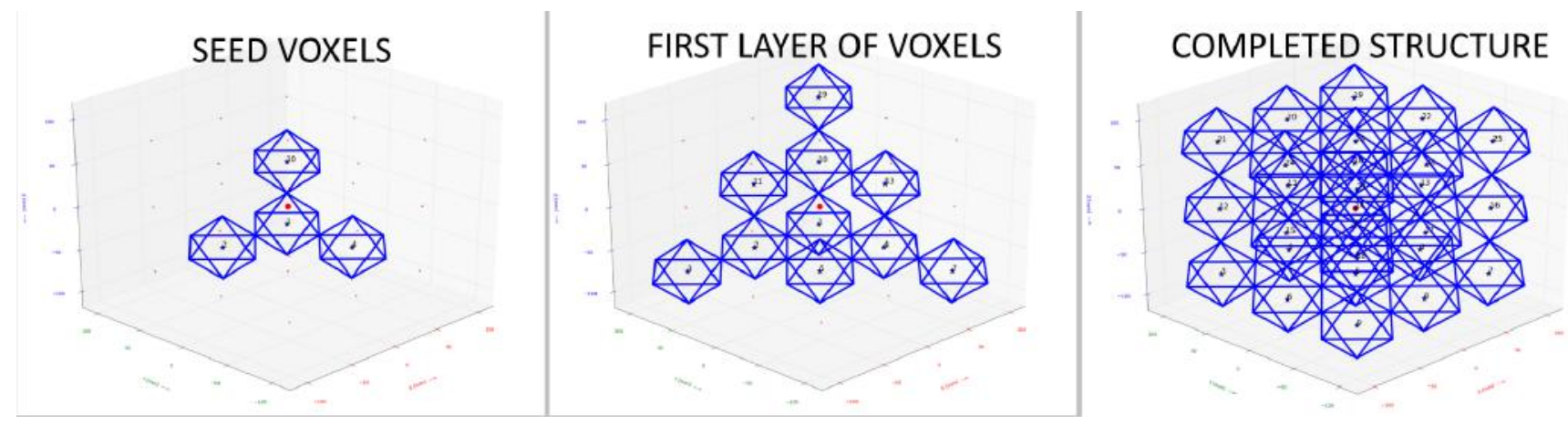

Figure 7. This figure shows an example of the path planning visualization at different steps to complete a $3 \times 3 \times 3$ structure.

The build path takes into account the voxel structure of interest and built tool limitations. In our case, the build tool is a robotic arm with end effector designed to build up structures diagonally outwards. Given this, the tool path is straight forward. A voxel structure is broken down into diagonal layers (on the $(1,1,1)$ plane) for which it will build incrementally in the $\mathrm{y}, \mathrm{x}$, and $\mathrm{z}$ directions sequentially. An example of a $3 \times 3 \times 3$ build is shown in Figure 7 . The figures shown are excerpts from visualizing the voxel structure build.

\section{RESULTS}

This section shows assembly time data from various cubic and beam structures constructed. The build speed of the system was set to a reasonable rate to ensure a safe working environment for development. We achieved an average part placement speed of about 1.5 voxels per minute or 1 voxel every 40 seconds, with a move speed of 3.15 inches per second. In a production environment, speeds can be increased significantly to optimize performance. Precision of the end effector was found to be critical. Table 1 and Figure 8 present the assembly time data for various lattice dimensions. The build process for a $2 \times 2 \times 2$ cube is shown in Figure 9.

Table 1. Assembly Times

\begin{tabular}{|c|c|c|}
\hline Structure & Number of Parts & Assembly Time (min.) \\
\hline \hline $2 \times 2 \times 2$ & 8 & 2.6 \\
$3 \times 3 \times 3$ & 27 & 15.3 \\
$3 \times 3 \times 3$ (hole) & 26 & 14.6 (expected) \\
$2 \times 2 \times 12$ & 48 & 57.5 (expected) \\
$3 \times 3 \times 12$ & 108 & 71.9 (expected) \\
\hline
\end{tabular}

\section{Discussion}

Implementation of the assembly system using a gantry system shows that this system could become a reliable, but slow of method of assembly. There were several aspects to the assembly that proved to be crucial to the building. We quickly realized that misalignment of the nodes could result in fastening errors where the bolt is driven in at an angle. This can be fixed in the future iterations with better fixture mechanisms. Our system was tolerant of placement errors up to about $2 \mathrm{~mm}$, but this will depend on the material of the voxel used. The build speed of the system could be increased, but we chose to stay at a reasonable speed to ensure a safe

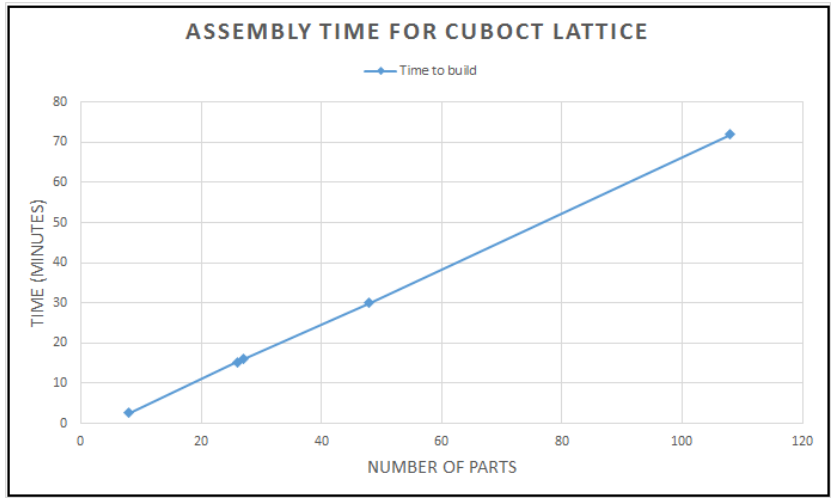

Figure 8. Plot of Number of Parts vs Time to build.

working environment. The end effector design also needs to be designed with higher precision in mind. Rotational errors during assembly could be extrapolated resulting in misplacement of the voxel.

In the future, feed systems will be incorporated directly into the gantry arm to improve upon build times. Although the demonstration was a success, further improvements in the control system as well as the fixturing mechanisms need to be refined to improve reliability.

The successful demonstration of assembling digital materials structure is a significant step in showing the feasibility of these systems in a larger ecosystem.

\section{Extrapolation}

We can describe the time required to build rectangular prism structures of any volume using an approximation based on shortest path distance in Equation 1.

$$
\sum_{x=1}^{n}\left[\sum_{y=1}^{n}\left[\sum_{z=1}^{n}\left(2 \sqrt{x^{2}+y^{2}+z^{2}}\right)\right]\right]
$$

For a cube structure, we can reduce the equation down to equations 2 and 3 . For a cube of side $\mathrm{n}$ elements, the time will scale by a factor of the 4th power when there is a pick-up station that it must travel back for part and hardware feeding. In Equation 3, the tool path distance is approximated as Manhattan distance, rather than Euclidean distance. The scaling factor reduces to the 3rd power when 


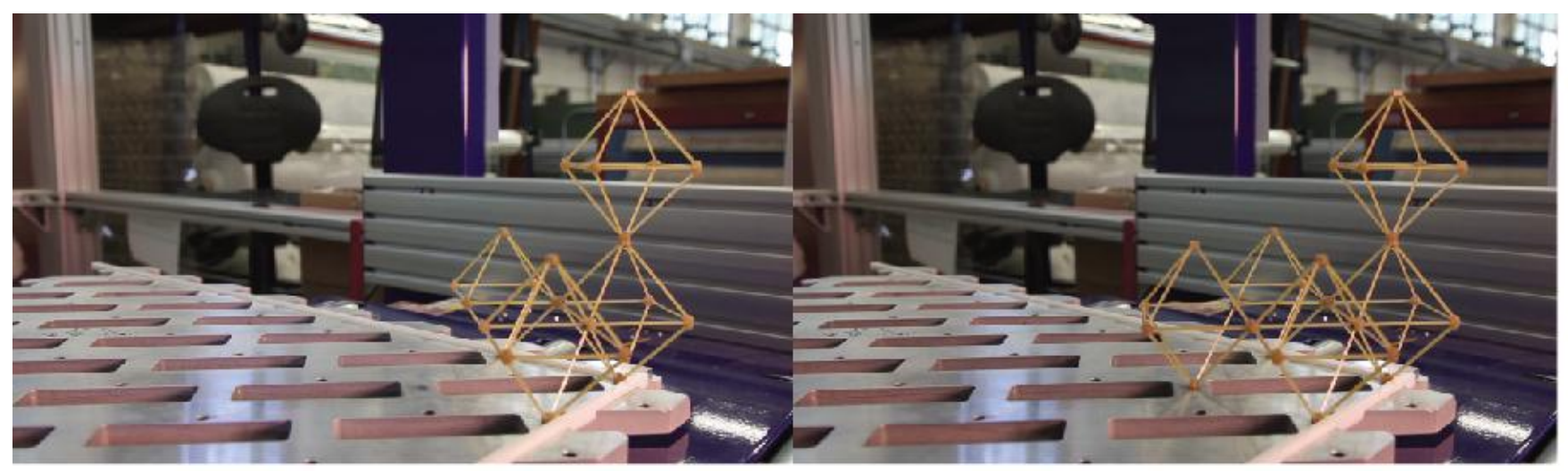

\section{Seed Voxels}

\section{1st Voxel Placed}
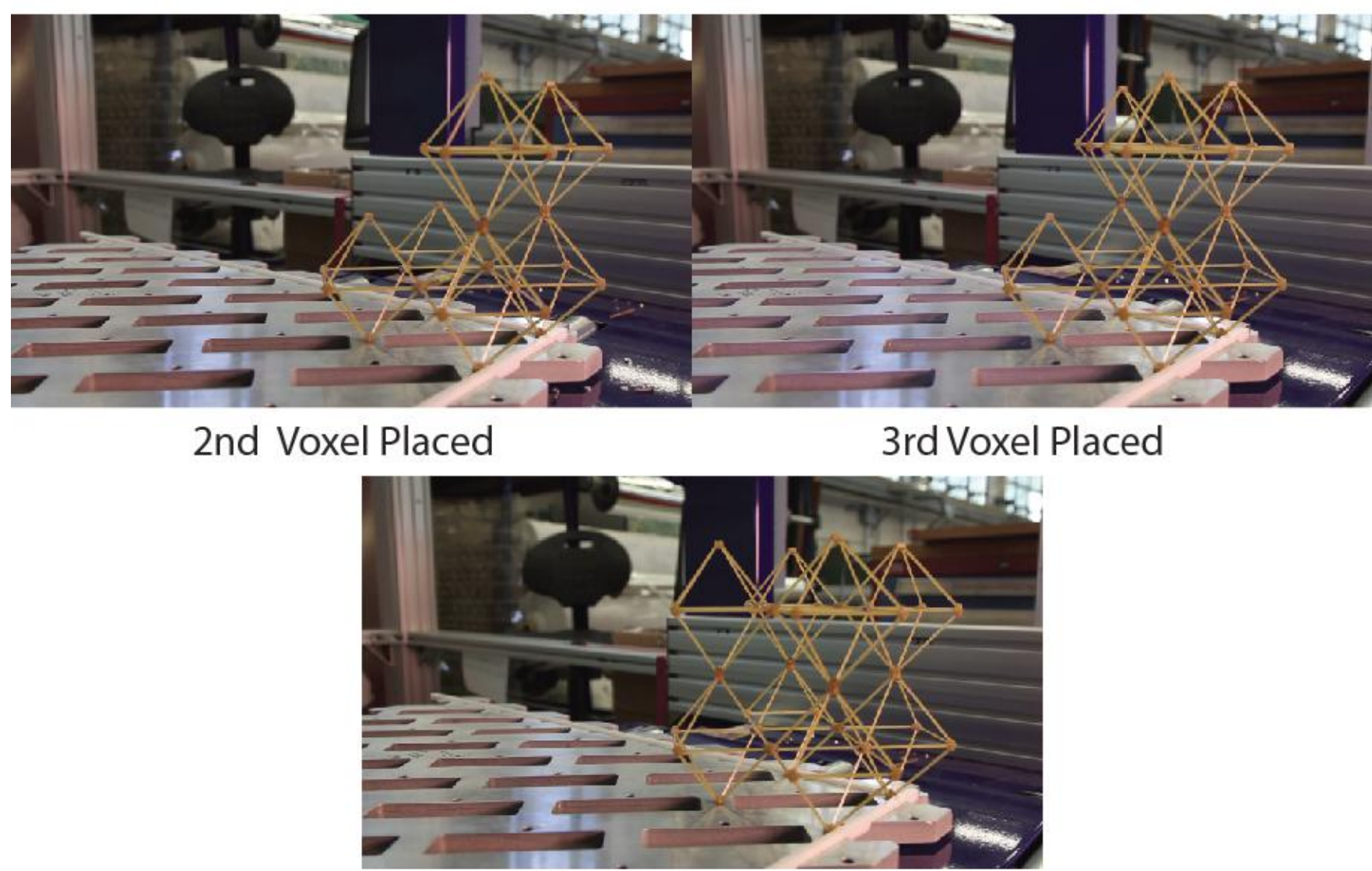

\section{4th Voxel Placed - 2×2×2 Cube Build}

Figure 9. This figure shows a photos of the build process for a $2 \times 2 \times 2$ cube.

feed mechanisms are build directly into the gantry machine. Note that at lower scales, the are variances in build times due to some voxel placement needing extra joints, but at larger scales they average out.

$$
\left[\sum_{x=1}^{n} x\right]\left[\sum_{y=1}^{n} y\right]\left[\sum_{z=1}^{n} z\right]=n^{3}
$$

$$
\begin{aligned}
\sum_{x=1}^{n}\left[\sum_{y=1}^{n}\left[\sum_{z=1}^{n} 2(x+y+z)\right]\right] & =3\left(n^{3}+n^{4}\right) \\
& =O\left(n^{4}\right)
\end{aligned}
$$

One of the important characteristics of the digital materials system is the ability to scale. In Table 2 we investigate scaling this type of assembly system with a $5 \times 5$ voxel beam extrusion from the $1 \mathrm{~m}$ scale up to the $10 \mathrm{~m}$ scale and $30 \mathrm{~m}$ scale. At the larger scales, the time to build becomes extraordinarily large. 
Implementation of parallelization, build in feed systems, and optimizations will be needed. Error correction will also needed to be accounted for because as build scale become larger, the errors will also increase [13].

Table 2. Build Scale Comparison

\begin{tabular}{|c|c|c|}
\hline Scale & Number of Voxels & Assembly Time (hours) \\
\hline \hline 1 m scale & 325 & 3.6 \\
10 m scale & 3,250 & 35.75 \\
30 m scale & 9,750 & 108.25 \\
\hline
\end{tabular}

\section{Comparison with other Assembly Systems}

Here we compare the build rate of the digital material systems to other assembly systems. One advantage of using digital materials is that the production rates will be the same no matter what material is used. Build speed can also be increased by using parallelization techniques. This is different from other extrusion techniques such as aluminum extrusions and thermoplastic extrusions, that rely on thermal properties for extrusion. Table 3 compares the CubOct Voxel extrusion to various other part placement speeds.

Table 3. Part Placement Comparison [6] [14]

\begin{tabular}{|c|c|c|}
\hline Type of Assy. & Part Placement & Extrusion Speed \\
\hline \hline CubOct Voxel & $1.5 \mathrm{parts} / \mathrm{min}$ & $.11 \mathrm{~m} / \mathrm{min}$ \\
LRC Truss & $.19 \mathrm{parts} / \mathrm{min}$ & $.38 \mathrm{~m} / \mathrm{min}$ \\
Aluminum Extru. & N/A & $5-80 \mathrm{~m} / \mathrm{min}$ \\
\hline
\end{tabular}

We can see that digital material as well as other ISA systems still need to much development before it can reach the level of current industrial production speeds. But with parallelization of the assembly, digital materials can theoretically match and/or exceed those speeds.

\section{Conclusion}

The study of digital materials is an exciting new field that combines materials science and robotics to to create high performing and reconfigurable structures. This work demonstrates the feasibility of assembling such systems and the potential of developing it in to an efficient assembly system. We are able to demonstrate a part placement speed of about 1.5 parts per minute. This shows that serial assembly techniques are too slow for a real production environment and improvements in this system must be implemented. This work showed that the assembly of such systems are sensitive to misalignment and precautions must be taken into account in future iterations. The techniques for assembly can be applied to future systems where parallelization and optimized feed mechanisms can help increase throughput.

\section{ACKNOWLEDGMENTS}

The authors thank the NASA ARMD Convergent Aeronautics Solutions (CAS) Program and NASA STMD GCD for funding this work. We also thanks Nick Cramer, Christine Gregg, and Irina Kostitsyna for critical discussions.

\section{REFERENCES}

[1] K. C. Cheung, "Digital Cellular Solids: reconfigurable composite materials," p. 147, 2012. [Online]. Available: http://dspace.mit.edu/handle/1721.1/78199

[2] K. C. Cheung and N. Gershenfeld, "Reversibly Assembled Cellular Composite Materials," 2013.

[3] M.-s. D. M. Modular and B. Jenett, "Meso-scale digital materials: modular, reconfigurable, lattice- based structures," pp. 1-11, 2016.

[4] B. Jenett, S. Calish, D. Cellucci, N. Cramer, N. Gershenfeld, S. Swei, and K. C. Cheung, "Digital Morphing Wing: Active Wing Shaping Concept Using Composite Lattice-based Cellular Structures,” 2016.

[5] J. Lymer, M. Hanson, A. Tadros, J. Boccio, B. Hollenstein, and K. Emerick, "Commercial Application of In-Space Assembly," American Institute of Aeronautics and Astronautics, pp. 1-17.

[6] M. D. Rhodes, R. W. Will, and C. C. Quach, "Verification tests of automated robotic assembly of space truss structures," Journal of Spacecraft and Rockets, vol. 32, no. 4, pp. 686-696, 1995. [Online]. Available: http://arc.aiaa.org/doi/abs/10.2514/3.26670

[7] E. Komendera, J. T. Dorsey, W. R. Doggett, and N. Correll, "Truss assembly and welding by Intelligent Precision Jigging Robots," in IEEE Conference on Technologies for Practical Robot Applications, TePRA, 2014.

[8] R. P. Hoyt, J. T. Slosad, T. J. Moser, J. I. Cushing, G. J. Jimmerson, R. L. Muhlbauer, A. J. Conley, S. R. Alvarado, and R. Dyer, "In-Space Manufacturing of Constructable Long-Baseline Sensors using the Trusselator Technology."

[9] B. Jenett and D. Cellucci, "A Mobile Robotic Platform for Exploration, Sensing, and Manipulation in a 3D Periodic Lattice Environment."

[10] N. Gershenfeld, M. Carney, B. Jenett, S. Calisch, and S. Wilson, "Macrofabrication with digital materials: Robotic assembly," Architectural Design, vol. 85, no. 5, pp. 122-127, 2015.

[11] M. Mikulas and D. J., "An Integrated In-Space Construction Facility for the 21st Century." NASA : Technical Memorandum 101515, 1988.

[12] M. Mikulas and H. Bush, "Mikulas Bush 1986 Space Station Assembled 5-m Struts.pdf," NASA : Technical Memorandum 89043, 1986.

[13] M. Carney and B. Jenett, "Relative Robots: Scaling Automated Assembly of Discrete Cellular Lattices," Proceedings of the 2016 Manufacturing Science and Engineering Conference, 2016.

[14] "Extrusion Process," http://www.aluminiumdesign.net/designsupport/extrusion-process/, accessed: 2016-10-30. 


\section{BIOGRAPHY}

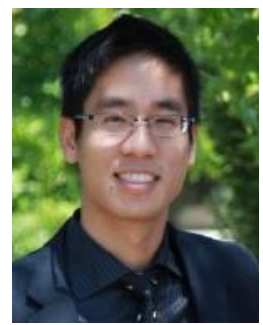

Greenfield Trinh is a research engineer in the Coded Structures Lab at NASA Ames Research Center. His current research activities include automated assembly of digital material structures and robotics. He received his B.S. in Physics from UCR and M.S. in Aerospace Engineering from San Jose State University.

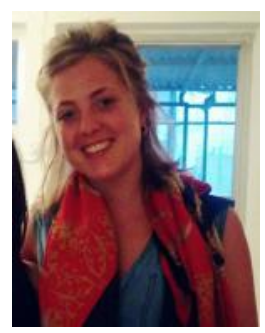

Grace Copplestone received a Masters in Engineering from the University of Cambridge, UK in 2014. She worked in robotics at Hanson Robotics in Hong Kong and NASA Ames Research Center, CA for a year before starting a Masters and Research Assistantship at the Center for Bits and Atoms in MIT.

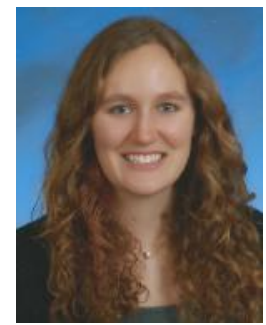

Molly O'Connor received a B.S. in Engineering Physics from Stanford University in 2015. Currently, she is pursuing her M.S. in Mechanical and Aerospace Engineering at UC Davis, where she is researching autonomous navigation for small satellites at the Center for Human/Robotics/Vehicle Integration and Performance. She interned with the NASA Ames Coded Structures Lab in 2016, working on automatic assembly of digital materials.

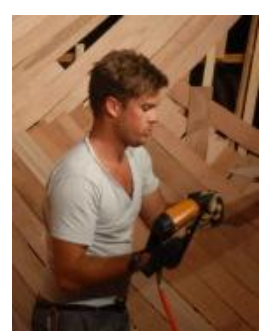

Benjamin Jenett is a Ph.D candidate from the Center for Bits and Atoms at the Massachusetts Institute of Technology. He conducts research on digital material structures and relative robots. He is also a NASA Space Technology Research Fellow. He drives a Miata.

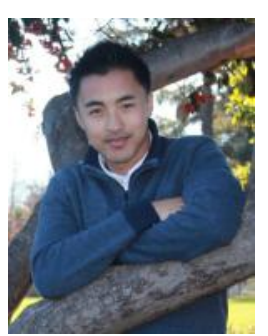

Steven Hu received a M.S. in Aerospace Engineering from Cal Poly, San Luis Obispo in 2007. He has been with NASA Ames for 3 years wherein he served various roles including software engineer, project manager and flight controller. Prior to Ames, he worked at JPL for 6 years as a systems engineer for various Earth Science flight projects.

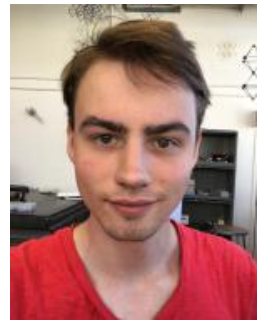

Sebastian Nowak is an undergraduate student studying Mechanical Engineering at Lehigh University. He has been working on Digital Material Assembly for the past four years, first at the Center for Bits and Atoms at MIT and now at NASA Ames. Currently he is developing a feeder mechanism for nuts and bolts to be used with a modified ShopBot for lattice assembly.

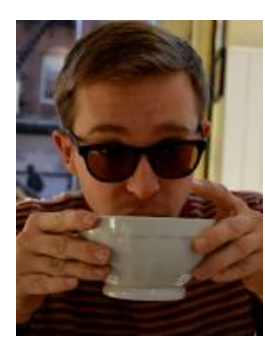

Daniel Cellucci is a Ph.D candidate studying Mechanical Engineering at Cornell University. He conducts research on digital material structures and relative robots. $\mathrm{He}$ is also a NASA Space Technology Research Fellow.

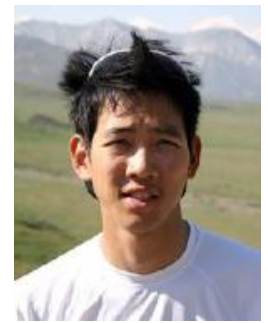

Kenneth Cheung received his Ph.D. from the Center for Bits and Atoms at the Massachusetts Institute of Technology. He helps to run the ARC Coded Structures Laboratory (CSL), which conducts research on the application of buildingblock based materials and algorithms to aeronautical and space systems. As a member of the Ames CCT staff, he materials and manufacturing. 